\title{
Rational Surfaces with Anticanonical Divisor not Reduced
}

\author{
Jesús A. Cerda Rodríguez, Gioia Failla, Mustapha Lahyane, Israel \\ Moreno-Mejía and Osvaldo Osuna-Castro
}

\begin{abstract}
We prove the finite generation of the monoid of effective divisor classes on a smooth projective rational surface $X$ endowed with an anticanonical divisor such that all its irreducible components are of multiplicity one except one which has multiplicity two. In almost all cases, the self-intersection of a canonical divisor $K_{X}$ on $X$ is strictly negative, hence $-K_{X}$ is neither ample nor numerically effective. In particular, $X$ is not a Del Pezzo surface. Furthermore, it is shown that the first cohomology group of a numerically effective divisor vanishes; as a consequence, we determine the dimension of the complete linear system associated to any given divisor on $X$.
\end{abstract}

\section{Introduction}

The aim of this work is to give an explicit construction of some smooth projective rational surfaces $X$ for which the monoid $M(X)$ of effective divisor classes modulo numerical equivalence is finitely generated and such that the complete linear system $\left|-K_{X}\right|$ of an anticanonical divisor $-K_{X}$ of $X$ contains only one element. Moreover, the first cohomology group of the invertible sheaf associated to any numerically effective divisor on $X$ vanishes. The ground field is assumed to be algebraically closed of arbitrary characteristic.

Key Words: Rational surfaces; Néron-Severi group; Blowing-up; Monoid of effective divisor classes.

2010 Mathematics Subject Classification: Primary 14J26; Secondary 14F05.

Received: January, 2012

Revised: March, 2013.

Accepted: April, 2013. 
A classical and very well studied class of rational surfaces is the one of Del Pezzo surfaces and standard examples are the ones provided by smooth cubic surfaces in the three dimensional projective space. Recall that the smooth projective surface $Z$ is a Del Pezzo if one of its anticanonical divisor $-K_{Z}$ is ample, that is, the self-intersection of $K_{Z}$ is greater than zero and the intersection number of $K_{Z}$ with any nonzero effective divisor is less than zero. In particular, a Del Pezzo surface is nothing than either $\mathbb{P}^{1} \times \mathbb{P}^{1}$ or a blowing up the projective plane $\mathbb{P}^{2}$ at $r$ points in general position with $r$ less than or equal to eight. It is worth to remind that the set of curves of strictly negative selfintersection on a Del Pezzo surface $Z$, not equal to $\mathbb{P}^{1} \times \mathbb{P}^{1}$ and $\mathbb{P}^{2}$, is reduced to the finite set of smooth projective rational curves of self-intersection equals -1 (these curves are usually called $(-1)$-curves) and generate the monoid of effective divisor classes on $Z$ modulo numerical equivalence, whereas the set of curves of strictly negative self-intersection on $\mathbb{P}^{1} \times \mathbb{P}^{1}$ or on $\mathbb{P}^{2}$ is empty. See the work by Brian Harbourne in [12] for a generalization of the notion of Del Pezzo surfaces.

From the work of Masayoshi Nagata (see [24, Theorem 4a, p. 283]), it is obvious that if $P_{1}, \ldots, P_{r}$ are points in the projective plane with $r$ greater than eight, one should specialize these points in order to ensure the finite generation of the monoid of effective divisor classes on the surface $Z$ obtained by blowing up the projective plane at $P_{1}, \ldots, P_{r}$. The case where $r$ is less than ten is studied in [25], [23], [15], [17], [18] and [19]. Some cases where $r$ is greater than nine have been studied in [10], [11], [14], [2], [5], [6], [7], [8], [9] and [21].

In ([22, Theorem (1.1), p. 271]), Eduard Looijenga studied smooth projective rational surfaces $S$ having an anticanonical divisor $-K_{S}$ of certain assigned type, but not allowing that some irreducible components of $-K_{S}$ to be multiple.

We are interested in providing rational surfaces $X$ with an anticanonical divisor $-K_{X}$ having two irreducible components one of which is of multipilicity two. The smooth projective rational surface $X=X_{(p, q)}$, where $p$ and $q$ are nonnegative integers, that we will deal with is constructed as follows. Take two distinct lines $L_{1}$ and $L_{2}$ in the projective plane $\mathbb{P}^{2}$ and consider $p$ points $P_{1}^{L_{1}}, \ldots, P_{p}^{L_{1}}$ of $L_{1}$ and $q$ points $P_{1}^{L_{2}}, \ldots, P_{q}^{L_{2}}$ of the degenerate conic $2 L_{2}$ such that the point of $L_{1}$ and $L_{2}$ does not belong to the set $\left\{P_{1}^{L_{1}}, \ldots, P_{p}^{L_{1}}, P_{1}^{L_{2}}, \ldots, P_{q}^{L_{2}}\right\}$. We allow $P_{i}^{L_{1}}$ (respectively $\left.P_{i}^{L_{2}}\right)$ to be infinitely near $P_{j}^{L_{1}}$ (respectively $P_{j}^{L_{2}}$ ) if $i$ is greater than $j$. The surface $X_{(p, q)}$ is nothing than the blow up the projective plane along its closed subscheme $\left\{P_{1}^{L_{1}}, \ldots, P_{p}^{L_{1}}, 2 P_{1}^{L_{2}}, \ldots, 2 P_{q}^{L_{2}}\right\}$.

From the classical Bézout Theorem (see Lemma 2.5 below), it follows that for almost all $p$ and $q$, the complete anticanonical linear system $\left|-K_{X_{(p, q)}}\right|$ is nonempty but with only one element. 
The following result asserts the finite generation of $M\left(X_{(p, q)}\right)$ for arbitrary nonnegative integers $p$ and $q$.

Theorem 1.1. With notation as above, the monoid $M\left(X_{(p, q)}\right)$ of effective divisor classes on $X_{(p, q)}$ modulo numerical equivalence is finitely generated.

As a consequence, the following result holds:

Corollary 1.2. With notation as above, the monoid of effective divisor classes modulo numerical equivalence $M\left(X_{(p, q)}\right)$ on $X_{(p, q)}$ is finitely generated by the set of smooth rational curves of strictly negative self-intersection.

As another consequence, the following known result is recovered, see [14, Lemma 3.1.1.]:

Corollary 1.3. The monoid of effective divisor classes on the surface obtained by the blow up the projective plane either at all collinear points or at the points of a degenerate conic whose support is a line is finitely generated.

The following lemma determine the numerically effective divisors which are orthogonal to $K_{X_{(p, q)}}$, its proof is postponed to Section 3. We recall that a divisor on $X_{(p, q)}$ is numerically effective if it meets every integral curve on $X_{(p, q)}$ nonnegatively.

Lemma 1.4. With the same notation as above, there is not nonzero numerically effective divisor $D$ on $X_{(p, q)}$, satisfying the equality $K_{X_{(p, q)}} . D=0$, $K_{X_{(p, q)}}$ being a canonical divisor on $X_{(p, q)}$.

Recall that for a divisor $C$ on a smooth projective surface $Y$ defined over a ground field $k$, and for every integer $i(i=0,1,2), h^{i}\left(Y, O_{Y}(C)\right)$ denotes the dimension over $k$ of the $i^{-t h}$ cohomology group $H^{i}\left(Y, O_{Y}(C)\right)$ of the natural sheaf $O_{Y}(C)$ associated to the divisor $C$. Here we study the vanishing problem of the first cohomology group of an arbitrary numerically effective divisor.

Theorem 1.5. With the same notation as above, if $D$ is a numerically effective divisor on $X_{(p, q)}$, then

$$
h^{1}\left(X_{(p, q)}, O_{X_{(p, q)}}(D)\right)=0,
$$

$O_{X_{(p, q)}}(D)$ being an invertible sheaf associated to the divisor $D$.

Proof. : Apply [13, Theorem III.1, p. 1197] and the above Lemma 1.7.

A straightforward consequence of Theorem 1.5 gives the dimension of the complete linear system of any numerically effective divisor on $X_{(p, q)}$. 
Corollary 1.6. With notation as above. Let $D$ be a numerically effective divisor on $X_{(p, q)}$ with $p q-p-q \leq 0$. Then

$$
h^{0}\left(X_{(p, q)}, O_{X_{(p, q)}}(D)\right)-1=\frac{1}{2}\left(D^{2}-D \cdot K_{X_{(p, q)}}\right)
$$

$O_{X_{(p, q)}}(D)$ being an invertible sheaf associated to the divisor $D$, and $K_{X_{(p, q)}}$ being a canonical divisor on $X_{(p, q)}$.

Proof. : Apply Theorem 1.5 and the below Lemmas 2.1 and 2.3 .

Corollary 1.7. With notation of as above. Let $D$ be a given divisor on $X_{(p, q)}$. Then $h^{0}\left(X_{(p, q)}, O_{X_{(p, q)}}(D)\right)$ is either equal to zero, to one or to

$$
1+\frac{1}{2}\left(M^{2}-M \cdot K_{X_{(p, q)}}\right),
$$

where $O_{X_{(p, q)}}(D)$ is an invertible sheaf associated to the divisor $D, M$ is the mobile part of the complete linear system $|D|$ and $K_{X_{(p, q)}}$ is a canonical divisor on $X_{(p, q)}$.

Proof. : Apply Corollary 1.6 to the numerically effective divisor $M$.

Remark 1.8. Points in the projective plane with the property that the surface obtained by blowing up these points satisfies the conclusion of Theorem 1.5 for every numerically effective divisor are called good points. See the works by Brian Harbourne in [11] and [12].

\section{Background on Rational Surfaces}

Let $Z$ be a smooth projective rational surface defined over an algebraically closed field of arbitrary characteristic. A canonical divisor on $Z$, respectively the Néron-Severi group $N S(Z)$ of $Z$ will be denoted by $K_{X}$ and $N S(X)$ respectively. There is an intersection form on $N S(X)$ induced by the intersection of divisors on $Z$, it will be denoted by a dot, that is, for $x$ and $y$ in $N S(Z)$, $x . y$ is the intersection number of $x$ and $y$ (see [16] and [1]).

The following result known as the Riemann-Roch theorem for smooth projective rational surfaces is stated using the Serre duality.

Lemma 2.1. Let $D$ be a divisor on a smooth projective rational surface $Z$ having an algebraically closed field of arbitrary characteristic as a ground field. Then the following equality holds:

$h^{0}\left(Z, O_{Z}(D)\right)-h^{1}\left(Z, O_{Z}(D)\right)+h^{0}\left(Z, O_{Z}\left(K_{Z}-D\right)\right)=1+\frac{1}{2}\left(D^{2}-D \cdot K_{Z}\right)$.

$O_{Z}(D)$ being an invertible sheaf associated to the divisor $D$. 
Here we recall some standard results, see [13] and [16]. A divisor class $x$ modulo numerical equivalence on a smooth projective rational surface $Z$ is effective respectively numerically effective, nef in short, if an element of $x$ is an effective, respectively numerically effective, divisor on $Z$. Here a divisor $D$ on $Z$ is nef if $D . C \geq 0$ for every integral curve $C$ on $Z$. Now, we start with some properties which follow from a successive iterations of blowing up closed points of a smooth projective rational surface.

Lemma 2.2. Let $\pi^{\star}: N S(X) \rightarrow N S(Y)$ be the natural group homomorphism on Néron-Severi groups induced by a given birational morphism $\pi: Y \rightarrow X$ of smooth projective rational surfaces. Then $\pi^{\star}$ is an injective intersectionform preserving map of free abelian groups of finite rank. Furthermore, it preserves the dimensions of cohomology groups, the effective divisor classes and the numerically effective divisor classes.

Proof. : See [13, Lemma II.1, p. 1193].

Lemma 2.3. Let $x$ be an element of the Néron-Severi group $N S(X)$ of a smooth projective rational surface $X$. The effectiveness or the the nefness of $x$ implies the noneffectiveness of $k_{X}-x$, where $k_{X}$ denotes the element of $\operatorname{Pic}(X)$ which contains a canonical divisor on $X$. Moreover, the nefness of $x$ implies also that the self-intersection of $x$ is greater than or equal to zero.

Proof. : See [13, Lemma II.2, p.1193].

The following result is also needed. We recall that a (-1)-curve, respectively a (-2)-curve, is a smooth rational curve of self-intersection -1 , respectively -2 .

Lemma 2.4. The monoid of effective divisor classes modulo algebraic equivalence on a smooth projective rational surface $X$ having an effective anticanonical divisor is finitely generated if and only if $X$ has only a finite number of $(-1)$-curves and only a finite number of $(-2)$-curves.

Proof. : See [20, Corollary 4.2, p. 109].

Finally, we recall the classical Bézout Theorem:

Lemma 2.5. Two projective plane curves of degree $\mu$ and $\nu$ defined over an algebraically closed ground field intersect in exactly $\mu \nu$ points, unless the curves have a common component. 


\section{Proof of Theorem 1.1 and Lemma 1.7}

In this section, we give a proof of the result stated in Theorem 1.1 of section one. To do so, we need to give explicitly the lattice structure of $N S\left(X_{(p, q)}\right)$. Firstly, the integral basis

$$
\left(\varepsilon_{0} ;-\varepsilon_{1}^{L_{1}}, \ldots,-\varepsilon_{p}^{L_{1}} ;-\varepsilon_{1}^{L_{2}}, \ldots,-\varepsilon_{q}^{L_{2}}\right),
$$

is defined by:

- $\varepsilon_{0}$ is the class of a line on the projective plane which do not pass through any of the assigned points $P_{1}^{L_{1}}, \ldots, P_{p}^{L_{1}} ; P_{1}^{L_{2}}, \ldots, P_{q}^{L_{2}}$ in consideration,

- $\mathcal{E}_{i}^{L_{1}}$ is the class of the exceptional divisor corresponding to the $i^{-t h}$ point blown-up $P_{i}^{L_{1}}$ for every $i=1, \ldots, p$,

- $\varepsilon_{j}^{L_{2}}$ is the class of the exceptional divisor corresponding to the $j^{-t h}$ point blown-up $P_{j}^{L_{2}}$ for every $j=1, \ldots, q$.

The class of a divisor on $X_{(p, q)}$ will be represented by the $(1+p+q)$-tuple

$$
\left(a ; b_{1}^{L_{1}}, \ldots, b_{p}^{L_{1}} ; b_{1}^{L_{2}}, \ldots, b_{q}^{L_{2}}\right)
$$

Secondly, the intersection form on $N S\left(X_{(p, q)}\right)$ is given by:

- $\varepsilon_{0}^{2}=1=-\left(\varepsilon_{i}^{L_{1}}\right)^{2}=-\left(\varepsilon_{j}^{L_{2}}\right)^{2}$ for every $i=1, \ldots, p$, and $j=1, \ldots, q$;

- $\mathcal{E}_{i}^{L_{1}} \cdot \mathcal{E}_{i^{\prime}}^{L_{1}}=0$ for every $i, i^{\prime}=1, \ldots, p$, with $i \neq i^{\prime}$;

- $\varepsilon_{j}^{L_{2}} \cdot \mathcal{E}_{j^{\prime}}^{L_{2}}=0$ for every $j, j^{\prime}=1, \ldots, q$, with $j \neq j^{\prime}$;

- $\varepsilon_{i}^{L_{1}} \cdot \varepsilon_{j}^{L_{2}}=0$ for every $i=1, \ldots, p, j=1, \ldots, q$;

- $\mathcal{E}_{0} \cdot \mathcal{E}_{i}^{L_{1}}=\mathcal{E}_{0} \cdot \mathcal{E}_{j}^{L_{2}}=0$ for every $i=1, \ldots, p, j=1, \ldots, q$.

Remark 3.1. we observe that if the class $\left(a ; b_{1}^{L_{1}}, \ldots, b_{p}^{L_{1}} ; b_{1}^{L_{2}}, \ldots, b_{q}^{L_{2}}\right)$ is effective, then it represents the class of a projective plane curve of degree a and having at least multiplicity $b_{1}^{L_{1}}, \ldots, b_{p}^{L_{1}}$ (respectively, $b_{1}^{L_{2}}, \ldots, b_{q}^{L_{2}}$ ) at the points $P_{1}^{L_{1}}, \ldots, P_{p}^{L_{1}}$ (respectively $\left.P_{1}^{L_{2}}, \ldots, P_{q}^{L_{2}}\right)$. Also we note by assumption that the classes $\mathcal{E}_{0}, \mathcal{E}_{i}^{L_{1}}, \mathcal{E}_{j}^{L_{2}}$ need not to be all the classes of smooth rational curves on $X_{(p, q)}$. 
From Lemma 2.4, we need to prove that the set of $(-1)$ and the set of $(-2)$-curves are both finite. To do so we first show that the set of $(-2)$-curves is finite. So let $N$ be a general $(-2)$-curve on $X_{(p, q)}$. Let $\pi$ be the natural projection from $X_{(p, q)}$ to $\mathbb{P}^{2}$ and let $\left(a ; b_{1}^{L_{1}}, \ldots, b_{p}^{L_{1}} ; b_{1}^{L_{2}}, \ldots, b_{q}^{L_{2}}\right)$ be the $(1+p+q)$ tuple representing the class of $N$ in the Néron-Severi group $N S\left(X_{(p, q)}\right)$ relative to the integral basis $\left(\varepsilon_{0} ;-\varepsilon_{1}^{L_{1}}, \ldots,-\varepsilon_{p}^{L_{1}} ;-\varepsilon_{1}^{L_{2}}, \ldots,-\varepsilon_{q}^{L_{2}}\right)$. Since $N^{2}=-2$, it follows that the degree $a$ is larger than one. From the two equalities $N^{2}=-2$ and $N \cdot K_{X_{(p, q)}}=0$, one may obtain the two following equalities:

$$
\left(b_{1}^{L_{1}}\right)^{2}+\ldots+\left(b_{p}^{L_{1}}\right)^{2}+\left(b_{1}^{L_{2}}\right)^{2}+\cdots+\left(b_{q}^{L_{2}}\right)^{2}=a^{2}+2,
$$

and

$$
\left(a-b_{1}^{L_{1}}-\cdots-b_{p}^{L_{1}}\right)+2\left(a-b_{1}^{L_{2}}+\cdots-b_{q}^{L_{2}}\right)=0 .
$$

Hence one may get.

$$
a-b_{1}^{L_{1}}-\cdots-b_{p}^{L_{1}}=0,
$$

and

$$
a-b_{1}^{L_{2}}+\cdots-b_{q}^{L_{2}}=0
$$

Now consider the new scalars $\left(\alpha_{i}^{L_{1}}\right)_{i \in\{1, \ldots, p\}}$ and $\left(\alpha_{j}^{L_{2}}\right)_{j \in\{1, \ldots, q\}}$ defined by:

$$
\alpha_{i}^{L_{1}}=b_{i}^{L_{1}}-\left(\frac{a}{p}\right), \text { for every } i=1, \ldots, p,
$$

and

$$
\alpha_{j}^{L_{2}}=b_{j}^{L_{2}}-\left(\frac{a}{q}\right), \text { for every } j=1, \ldots, q
$$

Then it follows from equations $3,4,15$ and 16 that:

$$
\alpha_{1}^{L_{1}}+\cdots+\alpha_{p}^{L_{1}}=0
$$

and

$$
\alpha_{1}^{L_{2}}+\cdots+\alpha_{p}^{L_{2}}=0 .
$$

The last two equalities in turn with the equation (1) give the following equality: 


$$
\left(\alpha_{1}^{L_{1}}\right)^{2}+\ldots+\left(\alpha_{p}^{L_{1}}\right)^{2}+\left(\alpha_{1}^{L_{2}}\right)^{2}+\cdots+\left(\alpha_{q}^{L_{2}}\right)^{2}=2+a^{2}-\left(\frac{a^{2}}{p}\right)-\left(\frac{a^{2}}{p}\right) .
$$

It therefore implies by assumption that $a$ is bounded.

Now we proceed to prove that the set of $(-1)$-curves on $X_{(p, q)}$ is finite. Indeed, let $E$ be a general (-1)-curve on $X_{(p, q)}$ and let $\left(a ; b_{1}^{L_{1}}, \ldots, b_{p}^{L_{1}} ; b_{1}^{L_{2}}, \ldots, b_{q}^{L_{2}}\right)$ be the $(1+p+q)$-tuple representing the class of $E$ in the Néron-Severi group $N S\left(X_{(p, q)}\right)$ relative to the integral basis $\left(\varepsilon_{0} ;-\varepsilon_{1}^{L_{1}}, \ldots,-\mathcal{E}_{p}^{L_{1}} ;-\varepsilon_{1}^{L_{2}}, \ldots,-\varepsilon_{q}^{L_{2}}\right)$. Since $E$ is general, it follows that the degree $a$ is greater than one. From the two equalities $E^{2}=-1$ and $E \cdot K_{S_{(p, q)}}=-1$, one may obtain the followings:

$$
\left(b_{1}^{L_{1}}\right)^{2}+\ldots+\left(b_{p}^{L_{1}}\right)^{2}+\left(b_{1}^{L_{2}}\right)^{2}+\ldots+\left(b_{q}^{L_{2}}\right)^{2}=a^{2}+1,
$$

and

$$
\left(a-b_{1}^{L_{1}}-\cdots-b_{p}^{L_{1}}-1\right)+2\left(a-b_{1}^{L_{2}}-\cdots-b_{q}^{L_{2}}\right)=0,
$$

Hence the only following case occurs:

$$
\left(b_{1}^{L_{1}}\right)^{2}+\ldots+\left(b_{p}^{L_{1}}\right)^{2}+\left(b_{1}^{L_{2}}\right)^{2}+\ldots+\left(b_{q}^{L_{2}}\right)^{2}=a^{2}+1,
$$

and

$$
\left(a-b_{1}^{L_{1}}-\cdots-b_{p}^{L_{1}}\right)=1,
$$

and

$$
\left(a-b_{1}^{L_{2}}-\cdots-b_{q}^{L_{2}}\right)=0,
$$

Now consider the new scalars $\left(\beta_{i}^{L_{1}}\right)_{i \in\{1, \ldots, p\}}$ and $\left(\beta_{j}^{L_{2}}\right)_{j \in\{1, \ldots, q\}}$ defined by:

$$
\beta_{i}^{L_{1}}=b_{i}^{L_{1}}-\left(\frac{a-1}{p}\right), \text { for every } i=1, \ldots, p,
$$

and

$$
\beta_{j}^{L_{2}}=b_{j}^{L_{2}}-\left(\frac{a}{q}\right), \text { for every } j=1, \ldots, q
$$

Hence the equation (10) gives rise to the following equality: 
$\left(\beta_{1}^{L_{1}}\right)^{2}+\ldots+\left(\beta_{p}^{L_{1}}\right)^{2}+\left(\beta_{1}^{L_{2}}\right)^{2}+\cdots+\left(\beta_{q}^{L_{2}}\right)^{2}=1+a^{2}-\left(\frac{(a-1)^{2}}{p}\right)-\left(\frac{a^{2}}{p}\right)$.

Consequently, by assumption $a$ is bounded and we are done.

Proof of Lemma 1.7.

Let $D$ be a numerically effective divisor on $X_{(p, q)}$ such that $D \cdot K_{X_{(p, q)}}=0$. We would like to prove that $D$ is the zero divisor. For let $\left(a ; b_{1}^{L_{1}}, \ldots, b_{p}^{L_{1}} ; b_{1}^{L_{2}}, \ldots, b_{q}^{L_{2}}\right)$ be the $(1+p+q)$-tuple representing the class of $D$ in the Néron-Severi group $N S\left(X_{(p, q)}\right)$ relative to the integral basis $\left(\varepsilon_{0} ;-\varepsilon_{1}^{L_{1}}, \ldots,-\mathcal{E}_{p}^{L_{1}} ;-\varepsilon_{1}^{L_{2}}, \ldots,-\mathcal{E}_{q}^{L_{2}}\right)$.

Let us prove that $a$ vanishes. Since $D \cdot K_{X_{(p, q)}}=0$, one may obtain the following equality.

$$
\left(a-b_{1}^{L_{1}}-\cdots-b_{p}^{L_{1}}\right)+2\left(a-b_{1}^{L_{2}}-\cdots-b_{q}^{L_{2}}\right)=0 .
$$

It follows that the following two equalities hold.

$$
a-b_{1}^{L_{1}}-\cdots-b_{p}^{L_{1}}=0 .
$$

and

$$
a-b_{1}^{L_{2}}-\cdots-b_{q}^{L_{2}}=0 .
$$

It follows by assumption and the fact that $D^{2} \geq 0$ that $a$ vanishes. Consequently, $D$ equals the zero divisor and we are done.

Acknowledgments. This research was partially supported by the projects CIC-UMSNH 2010-2013, PAPIIT IN102008, and PROMEP UMSNH-PTC214."

\section{References}

[1] W. Barth, C. Peters, A. Van de Ven, Compact Complex Surfaces, Berlin, Springer 1984.

[2] A. Campillo, G. González-Sprinberg, On characteristic cones, clusters and chains of infinitely near points, in: Brieskorn Conference Volume, in: Progr. Math., vol. 162, Birkhäuser, 1998. 
[3] A. Campillo, O. Piltant, A. Reguera, Cones of Curves and of Line Bundles on Surfaces Associated with Curves Having one Point at Infinity, Proceedings of the London Mathematical Society, Vol.84, Issue 03 (2002), 559-580.

[4] A. Campillo, O. Piltant, A. Reguera, Cones of curves and of line bundles at infinity. Journal of Algebra 293, 503-542 (2005).

[5] C. Galindo, F. Monserrat, The cone of curves associated to a plane configuration, Commentarii Mathematici Helvetici 80 (2005), 75-93.

[6] C. Galindo, F. Monserrat, The cone of curves of Line Bundles of a Rational Surface, International Journal of Mathematics. Vol. 15, No. 4 (2004), 393-407.

[7] G. Failla, M. Lahyane, G. Molica Bisci, On the finite generation of the monoid of effective divisor classes on rational surfaces of type $(n, m)$, Atti dell' Accademia Peloritana dei Pericolanti Classe di Scienze Fisiche, Matematiche e Naturali Vol. LXXXIV, C1A0601001 (2006)

[8] G. Failla, M. Lahyane, G. Molica Bisci, The finite generation of the monoid of effective divisor classes on Platonic rational surfaces. Singularity Theory, Proceedings of the 2005 Marseille Singularity School and Conference, 565-576, CIRM, Marseille, France. World Scientific Publishing Co. 2007.

[9] G. Failla, M. Lahyane, G. Molica Bisci, Rational Surfaces of Kodaira type $I V$, Bollettino dell'Unione Matematica Italiana, Sezione B; articoli di ricerca matematica, Volume 10, Number 3 (2007), pages 741 - 750.

[10] B. Harbourne, Blowings-up of $\mathbb{P}^{2}$ and their blowings-down, Duke Mathematical Journal, 52:1 (1985), 129 - 148.

[11] B. Harbourne, Points in good position in $\mathbb{P}^{2}$, Zero-dimensional schemes, Proceedings of the International Conference held in Ravello, Italy, June 8-13, 1992, De Gruyter, Berlin, 1994.

[12] B. Harbourne, Rational surfaces with $K^{2}>0$, Proceedings of the American Mathematical Society, Volume 124 Number 3 (March 1996), 727 733.

[13] B. Harbourne, Anticanonical rational surfaces, Transactions of the American Mathematical Society, Volume 349 Number 3 (1997), 1191 - 1208.

[14] B. Harbourne, Free resolutions of fat point ideals on $\mathbb{P}^{2}$, Journal of Pure and Applied Algebra, 125 (1998), 213 - 234. 
[15] B. Harbourne, R. Miranda, Exceptional curves on rational numerically elliptic surfaces, Journal of Algebra, 128 (1990), 405 - 433.

[16] R. Hartshorne, Algebraic Geometry, Graduate Texts in Mathematics, Springer Verlag (1977).

[17] M. Lahyane, Exceptional curves on rational surfaces having $K^{2} \geq 0$, Comptes Rendus Mathematique, Volume 338, Issue 11, 1 June 2004, 873 -878 .

[18] M. Lahyane, Rational surfaces having only a finite number of exceptional curves, Mathematische Zeitschrift, Volume 247 Number 1 (May 2004), $213-221$.

[19] M. Lahyane, Exceptional curves on smooth rational surfaces with $-K$ not nef and of self-intersection zero, Proceedings of the American Mathematical Society, 133 (2005), 1593 - 1599.

[20] M. Lahyane, B. Harbourne, Irreducibility of -1-classes on anticanonical rational surfaces and finite generation of the effective monoid, Pacific Journal of Mathematics, Volume 218 Number 1 (2005), 101 - 114.

[21] M. Lahyane, On the finite generation of the effective monoid of rational surfaces, Journal of Pure and Applied Algebra, Volume 214, Issue 7 (2010), $1217-1240$.

[22] E. Looijenga, Rational surfaces with an anticanonical cycle, Annals of Mathematics, 114 no. 2 (1981), 267 - 322.

[23] R. Miranda, U. Persson, On extremal rational elliptic surfaces, Mathematische Zeitschrift, 193 (1986), 537 - 558.

[24] M. Nagata, On rational surfaces, II, Memoirs of the College of Science, University of Kyoto, Series A 33 (1960), no. 2, 271 - 293.

[25] J. Rosoff, Effective divisor classes and blowings-up of $\mathbb{P}^{2}$, Pacific Journal of Mathematics, 89 (2) (1980), 419 - 429. 
Jesús Adrian Cerda Rodríguez, Mustapha Lahyane, Osvaldo Osuna-Castro

Instituto de Física y Matemáticas (I.F.M.)

Universidad Michoacana de San Nicolás de Hidalgo (U.M.S.N.H.)

Edificio C-3, Ciudad Universitaria.

C. P.58040 Morelia, Michoacán, México.

E-mail: \{jacerda,lahyane,osvaldo\}@ifm.umich.mx

Gioia Failla

University of Reggio Calabria, DIIES,

Via Graziella, Feo di Vito,

Reggio Calabria, Italy

E-mail:gioia.failla@unirc.it

Israel Moreno-Mejía

Instituto de Matemáticas,

Universidad Nacional Autónoma de México,

México, D.F., C.P.04510

E-mail: israel@matem.unam.mx 